\title{
Energy Efficiency and Capacity Tradeoff in Cloud Radio Access Network of High-Speed Railways
}

\author{
Shichao Li, ${ }^{1,2}$ Gang Zhu, ${ }^{1,2}$ Siyu Lin,, ${ }^{1,2}$ Qian Gao, ${ }^{1,2}$ Lei Xiong, \\ Weiliang Xie, ${ }^{3}$ and Xiaoyu Qiao ${ }^{3}$ \\ ${ }^{1}$ State Key Laboratory of Rail Traffic Control and Safety, Beijing Jiaotong University, Beijing 100044, China \\ ${ }^{2}$ School of Electronic Information Engineering, Beijing Jiaotong University, Beijing 100044, China \\ ${ }^{3}$ Technology Innovation Center, China Telecom, Beijing 100000, China \\ Correspondence should be addressed to Siyu Lin; sylin@bjtu.edu.cn
}

Received 29 July 2016; Revised 23 November 2016; Accepted 18 December 2016; Published 9 January 2017

Academic Editor: Piotr Zwierzykowski

Copyright (C) 2017 Shichao Li et al. This is an open access article distributed under the Creative Commons Attribution License, which permits unrestricted use, distribution, and reproduction in any medium, provided the original work is properly cited.

\begin{abstract}
To meet the increasing demand of high-data-rate services of high-speed railway (HSR) passengers, cloud radio access network (C-RAN) is proposed. This paper investigates the tradeoff between energy efficiency (EE) performance and capacity in C-RAN of HSR. Considering that the train location can be predicted, we propose a predictable path loss based time domain power allocation method (PPTPA) to improve EE performance of HSR communication system. First, we consider that the communication system of HSR only bears the passenger information services (PISs). The energy-efficient power allocation problem with delay constraint is studied. The formulated problem is nonconvex. To deal with it, an equivalent convex problem is reformulated. Based on PPTPA, we propose an iterative algorithm to improve the EE performance. Second, we consider that the PISs and the train control services (TCSs) are all bore. A capacity optimization problem with joint EE and services transmission delay constraints is formulated. Based on PPTPA, we propose a hybrid power allocation scheme to improve the capacity of the system. Finally, we analyze the effect of small-scale fading on EE performance. The effectiveness of the proposed power allocation algorithm is validated by HSR channel measurement trace based emulation results and extensive simulation results.
\end{abstract}

\section{Introduction}

In the past couple of years, high-speed railways (HSR) are expanding rapidly all over the world. More than $16,000 \mathrm{~km}$ HSR are deployed in China, which accounts for more than 60 percent of operation length of HSR around the world. The railway communication system plays a key role in HSR to bear the train control services (TCSs) and passenger information services (PISs) $[1,2]$.

Although current mobile communication technologies can guarantee the safety of HSR as bearing the TCSs, the relatively low transmission data rate (e.g., 2-4 Mbps) cannot provide satisfied quality of experience (QoE) of passengers. Therefore, the mobile communication system with distributed antennas is implemented in HSR scenario to improve the passengers' QoE [3]. Distributed antenna system (DAS) is consisted with radio remote units (RRUs) and base stations (BSs), but the radio resource cannot be shared between the RRUs, which limits the centralized processing gain of DAS. To mitigate this issue, cloud radio access network (CRAN) is proposed based on DAS structure $[4,5]$, in which a baseband unit (BBU) pool is instead of the distributed deployed BSs to handle the complex computational tasks. The centralized signal processing structure in the BBU pool has much more advantages than distributed signal processing in separate BSs, including saving the power, reducing the operating expenditure, and improving hardware utilization ratio. C-RAN changes the static relationship between BBU and RRUs, which is by now recognized as a good solution to provide high-speed data services to meet the increasing demand of high-data-rate services of HSR passengers [6, 7]. In addition, C-RAN can reduce the handover frequency of the train. Due to the high movement speed of the trains, the handover happens frequently. C-RAN can provide extensive radio coverage via super cell to limit the handover frequency 
and the $\mathrm{BBU}$ pool can eliminate interference to improve the coverage quality [3].

As energy consumption for communication technologies has been growing rapidly, a large number of greenhouse gases are discharged [8]. Recent reports suggest that $2 \%$ of the world-wide $\mathrm{CO}_{2}$ is discharged by communication infrastructures and 3\% of the world-wide energy is consumed [9]. For the rail transportation system, around $50 \%$ of the energy is consumed by trains, and the rest is used by infrastructure facilities (stations, communication equipment, groundwater pumps, tunnel lighting, etc.) to ensure the proper system operation [10, 11]. As we know, HSR is a kind of green transportation system with low energy consumption, in order to save energy, all the infrastructure facilities need to improve EE performance. For the communication equipment of HSR, the BSs are deployed densely along the railway line ( 2 base stations are deployed each 3 kilometers). For example, there are about 900 BSs in Beijing-Shanghai HSR line. Therefore, how to utilize the limited energy to meet the needs of growing communication traffic is a significant problem [12-15].

Resource allocation in the C-RAN has attracted considerable attention in recent years. To maximize the sum capacity in C-RAN system, Zhou and Yu adapted Wyner-Ziv coding method to enhance the performance of C-RAN [16]. To reduce the energy consumption of C-RAN, a joint power minimization and RRUs selection problem were formulated [17]. Comparing with saving energy, how to improve the capacity with unit power is a more practical problem, so EE is an important performance metric in wireless communication system design. There are several EE performance-improving methods in C-RAN, such as joint distributed compression in the uplink [18] and joint power and subcarriers allocation in the downlink [19].

To pursue the EE maximization, the system capacity may degrade dramatically [20], so the tradeoff between capacity and $\mathrm{EE}$ is worth investigating. An effective EE constrained rate optimal power allocation policy for Nakagami-m channels was proposed in [20]. To maximize the EE performance in a distributed antenna system subject to the users' quality of service (QoS), backhaul capacity and antenna transmission power, a joint antenna, subcarrier, and power allocation method were proposed [20]. A joint antenna, subcarrier, and power allocation method was proposed [21]. In order to improve the EE performance of data center networks, a mechanism via the elastic multicontroller software defined networks was proposed [22]. Wu et al. proposed a method to improve the EE performance when considering multiple users harvest energy from a power station and then communicate with an information station in a time-division manner [23]. A convex relaxation and global optimization method was proposed to improve the EE performance in multiuser multicarrier broadband wireless systems [24]. There are also some works about the balance between the capacity and EE performance; for example, $\mathrm{Ng}$ et al. proposed an iterative resource allocation policy which considered the tradeoff between network capacity, EE performance, and backhaul capacity in multicell networks [25]. All the previous works are designed for conventional cellular networks.
Since C-RAN can provide various high-speed wireless services for users, it can be deployed along the high-speed lines to improve the QoE of passengers. As HSR is a kind of green transportation system with low energy consumption, the energy consumption of mobile communication system in HSR also needs to be considered [26]. All the existing works about EE optimization of C-RAN are investigated in the conventional cellular networks. Therefore, how to improve the EE performance in HSR considering the HSR characteristic is an practical interesting problem. Generally, resource allocation requires the accurate channel state information (CSI). Comparing with the conventional cellular networks, it is hard to estimate the accurate CSI in HSR scenario. However, due to the line of sight (LoS) scenario and predicted location of train in HSR system, the CSI can be simplified as predicted path loss information, which can be estimated via location information of the train.

There are two major concerns in the EE optimization in HSR. First, the QoE requirements of passengers should be considered. For the C-RAN structure, complex computational tasks are done by Virtual Machines (VMs) in BBU pool, which introduces extra latency to the service transmission. To achieve the delay and transmission rate requirements of passengers, the processing time of VMs should be considered in the EE optimization of C-RAN. Second, the communication system of HSR bears not only the PISs but also the TCSs. To pursue the EE maximization, the system capacity may degrade dramatically [20], which is inconsistent with the high system capacity requirement. Therefore, the tradeoff between EE performance and capacity should be considered.

In this paper, considering the train location can be predicted, we propose a predictable path loss based time domain power allocation method (PPTPA) to improve EE performance of HSR communication system. First, we only consider the communication system of HSR bearing the PISs. We focus on the EE optimization problem with delay constraint of C-RAN in the HSR scenario. The VMs processing latency in the $\mathrm{BBU}$ pool and radio transmission delay of services are considered in the proposed power allocation scheme to maximize EE performance in C-RAN. Since the EE maximization formulation is a nonconvex problem, we reformulate the objective function. Based on PPTPA, we propose an iterative algorithm to improve the EE performance. Second, we consider the communication system of HSR bearing the PISs and the TCSs. A capacity optimization problem subject to joint $\mathrm{EE}$ requirement and services transmission delay constraints is formulated. Based on PPTPA, we propose a hybrid power allocation scheme to improve the capacity of the system. Finally, the effects of small-scale fading on the proposed power allocation scheme performance are evaluated. Zhengzhou-Xian HSR line channel measurements trace based emulation results and extensive simulation results are provided to validate that the proposed two power allocation policies can meet the QoE requirements of PISs and TCSs.

The rest of the paper is organized as follows. The system model is described in Section 2. In Section 3, we analyze power allocation problem with services transmission delay constraint and utilize PPTPA to maximize EE performance. In Section 4, we investigate the optimal power allocation to 


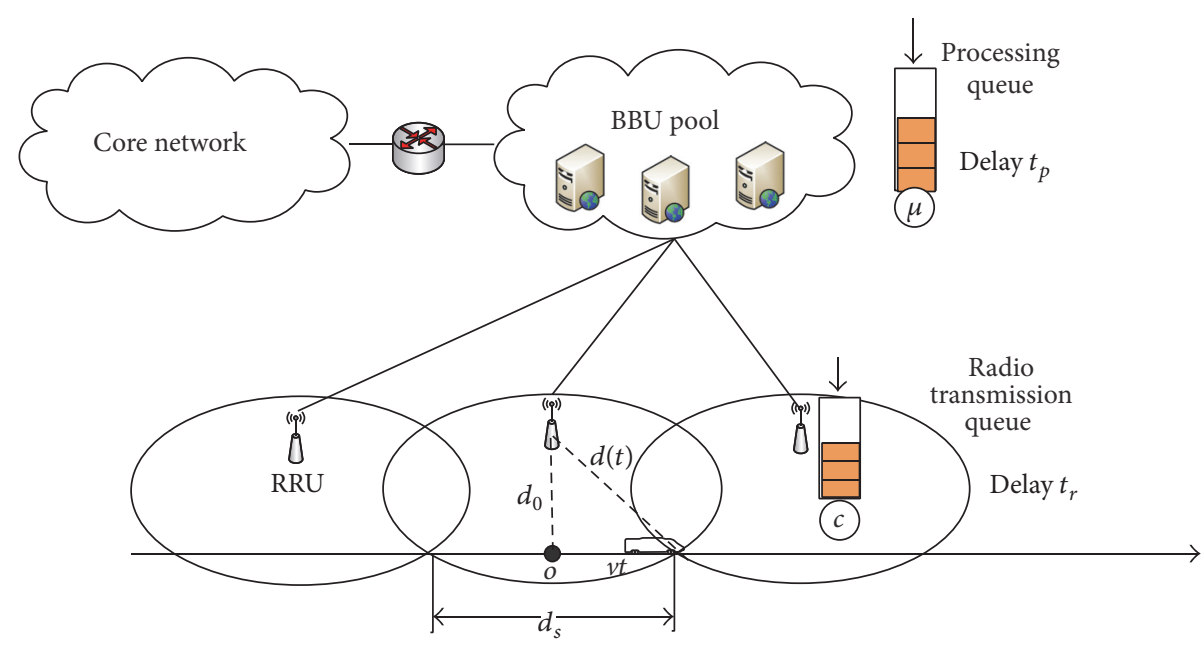

FIGURE 1: System model.

maximize capacity with joint services transmission delay and EE constraints. In Section 5, emulation results and extensive simulation results are provided to justify our analysis. Conclusion is given in Section 6.

\section{System Model}

The C-RAN under consideration consists of a set of RRUs and a BBU pool as shown in Figure 1. The RRUs are deployed along the railway line to provide the radio coverage. In this work, we only focus on the downlink of C-RAN, as it is always the bottleneck of the mobile communication system. The downlink traffic transmission can be divided into two phases, one is data preprocessing in the BBU pool and the other is radio transmission by the RRUs. The two phases can be modeled as two queue models, respectively [27]. One is the service data preprocessing queue, in which the data packets are preprocessed (such as encoded) by the VMs of $\mathrm{BBU}$ pool. The processing rate of $\mathrm{VMs}$ in $\mathrm{BBU}$ pool is denoted as $\mu$, which can be considered as the service rate in the service data preprocessing queue. The other queue is the radio transmission queue, in which the service data are transmitted from the RRUs to the passengers packet by packet. The radio transmission rate is denoted as $c$, which can be considered as service rate in the radio transmission queue. The BBU pool connects RRUs via fiber links, so the transmission delay of these links can be ignored. We assume that the service arrival process from core network is a Poisson process with mean rate $\lambda$. The service time in the preprocessing queue follows exponential distribution with mean $1 / \mu$ and the service time of the radio transmission queue follows exponential distribution with mean $1 / c$.

In HSR scenario, most of the services are sensitive to delay, that is, video on demand and voice services. Therefore, delay is a key metric to measure QoE. The transmission delay of the traffic in the C-RAN should be less than the QoE requirement of passengers. In this system, $t_{p}=1 /(\mu-\lambda)$ is denoted as the data processing delay and $t_{r}=1 /(c-\lambda)$ is denoted as the radio transmission delay. $t_{t}$ is the expected delay in this system, which can be expressed as $t_{t}=t_{p}+t_{r}=$ $1 /(\mu-\lambda)+1 /(c-\lambda)$, for $\mu>\lambda, c>\lambda$. The transmission delay requirement can be described as

$$
t_{t} \leq \tau
$$

where $\tau$ is the transmission delay requirement of passengers. If the service is HTTP/web (emails), it can be represented as a new service with different QoE requirements. As the HTTP/web (emails) requires low data rate and loose delay, we can adjust the delay constraint of QoE requirement.

The coverage model of RRUs is shown in Figure 1, the distance between RRU and railway line is $d_{0}$, and the coverage diameter of each RRU is $d_{s}$. The train speed is $v$ and the period of the train crossing one cell covered by RRU is $T=d_{s} / v$. RRUs can eliminate the interinterference by BBU pool, so we do not consider the interference between RRUs [28].

\section{EE Maximization Problem}

Due to the rapid increase of the operating cost of wireless communication system and carbon emission, the EE performance has become a main issue for the design of future wireless communication system [26]. In this section, we formulate the EE maximization with QoE provisioning problem firstly. As the objective function is a nonlinear fractional form, the objective function is reformulated, and then an iterative algorithm based on PPTPA is proposed to solve this problem.

3.1. Problem Formulation. To guarantee the QoE requirement of passengers, the transmission delay should be satisfied. Constant power allocation policy cannot meet the transmission delay requirement due to the varying fading channel [29]. Therefore, we adopt variable transmit power allocation policy. In this section, our objective is to maximize EE performance, and the EE performance can be defined as $C / P$ bits/joule [3].

Let $t=0$, when the train arrives at the point $O$ as shown in Figure 1, $h(t)$ denotes the channel gain at time $t$. In HSR 
scenario, it is difficult to get the accurate CSI of the train. However, due to the moving track of the train being certain, the predicted path loss information instead of accurate CSI is used for resource allocation. The path loss is described as

$$
h(t)=\frac{1}{\left(d_{0}^{2}+(v t)^{2}\right)^{\alpha / 2}}+\Delta, \quad-\frac{d_{s}}{2 v} \leq t \leq \frac{d_{s}}{2 v},
$$

where $\alpha$ is the path loss exponent and $\Delta$ is a constant related to the height of the transmit antenna and the frequency. Because the channel variation is periodical from one cell to another and that is symmetrical in one cell, we only consider half of a period of the channel variation to design power allocation policy.

We denote $\mathscr{P}$ as a power allocation policy, and the corresponding channel capacity can be calculated as

$$
C(t)=\log (1+h(t) \mathscr{P}(h(t)))
$$

Energy consumption plays an important role in greenhouse gas emissions, so a high EE performance is a main concern point for the design of future wireless communication. In addition, delay is a key metric to measure QoE. In this paper, our aim is to maximize the EE performance from RRUs to the passengers with the constraints of radio transmission rate, transmission delay requirement, and average power. Inequation (1) is transmission delay requirement. From (1), we can get $c \geq \lambda+1 / \tau+1 /\left(\tau^{2}(\mu-\lambda)-\tau\right)$.

Problem 1 (EE maximization problem). Considering the transmission delay constraint and average power constraint, the EE maximization problem in the downlink C-RAN of HSR is formulated as

$$
\begin{array}{ll}
\text { (P1) } \max _{\mathscr{P}} \frac{\int_{0}^{T / 2} C(t) d t}{\int_{0}^{T / 2} \mathscr{P}(h(t)) d t+P_{c}} \\
\text { subject to } & \frac{2}{T} \int_{0}^{T / 2} C(t) d t \geq c, \\
& c \geq \lambda+\frac{1}{\tau}+\frac{1}{\tau^{2}(\mu-\lambda)-\tau}, \\
& \frac{2}{T} \int_{0}^{T / 2} \mathscr{P}(h(t)) d t \geq P_{\text {ave }},
\end{array}
$$

where $P_{c}$ is the circuit power consumption and $P_{\text {ave }}$ is average transmission power of RRU. Constraint (4b) means that the average channel capacity should be large or equal to the radio transmission rate in half of a period. Constraint (4c) corresponds to the transmission delay requirement. Constraint (4d) means the average power constraint of RRU in half of a period.

Constraint (4b) is a concave function; (4c) and (4d) are linear functions. Therefore, (4b), (4c), and (4d) are all convex constraints. But the objective function is a nonlinear fractional programming, so Problem 1 is a nonconvex optimization problem [30].

3.2. EE Maximization Problem Reformulation. Problem 1 is a nonconvex optimization problem, so it cannot be solved by classical convex optimization methods. In this section, we reformulate the objective function of Problem 1 .

We define a nonnegative variable $\gamma^{*}$ as the energyefficient optimal value $\gamma^{*}=\int_{0}^{T / 2} C^{*}(t) d t / \int_{0}^{T / 2} \mathscr{P}^{*}(h(t)) d t+$ $P_{c}$, where $\mathscr{P}^{*}(h(t))$ is the optimal power allocation policy and $C^{*}(t)$ is the corresponding channel capacity with the optimal power allocation policy [31].

Lemma 2. $\gamma^{*}$ can be achieved if and only if

$$
\begin{aligned}
\max _{\mathscr{P}} & \int_{0}^{T / 2} C(t) d t-\gamma^{*}\left(\int_{0}^{T / 2} \mathscr{P}(h(t)) d t+P_{c}\right) \\
= & \int_{0}^{T / 2} C^{*}(t) d t \\
& -\gamma^{*}\left(\int_{0}^{T / 2} \mathscr{P}^{*}(h(t)) d t+P_{c}\right)=0 .
\end{aligned}
$$

Proof. The proof of Lemma 2 is in the Appendix.

From Lemma 2, if we can find the energy-efficient optimal value $\gamma^{*}$, Problem 1 can be solved. However, $\gamma^{*}$ cannot be calculated directly; we propose an iterative algorithm (Algorithm 1) to update $\gamma$ while ensuring the corresponding solution $\mathscr{P}(h(t))$ remains feasible in each iteration. Then, optimal resource allocation policy to solve Problem 3 can be derived.

Problem 3 (reformulated EE maximization problem).

$$
\begin{aligned}
& \text { (P3) } \max _{\mathscr{P}} \int_{0}^{T / 2} C(t) d t \\
& -\gamma^{*}\left(\int_{0}^{T / 2} \mathscr{P}(h(t)) d t+P_{c}\right) \\
& \text { subject to } \frac{2}{T} \int_{0}^{T / 2} C(t) d t \geq \lambda+\frac{1}{\tau}+\frac{1}{\tau^{2}(\mu-\lambda)-\tau} \text {, } \\
& \frac{2}{T} \int_{0}^{T / 2} \mathscr{P}(h(t)) d t \leq P_{\text {ave }}
\end{aligned}
$$

where $\gamma^{*}$ is the energy-efficient optimal value.

3.3. Proposed Iterative Algorithm. For Problem 3, we propose an iterative algorithm to update $\gamma$ as Algorithm 1. The outer loop can update $\gamma^{(i+1)}$ through $C^{(i)}(t)$ and $\mathscr{P}^{(i)}(h(t))$ of each iteration. The inner loop can calculate the power allocation policy of $\mathscr{P}^{(i)}(h(t))$ and $C^{(i)}(t)$ by using the Lagrangian dual method. The outer loop can calculate $\gamma^{(i)}$. 


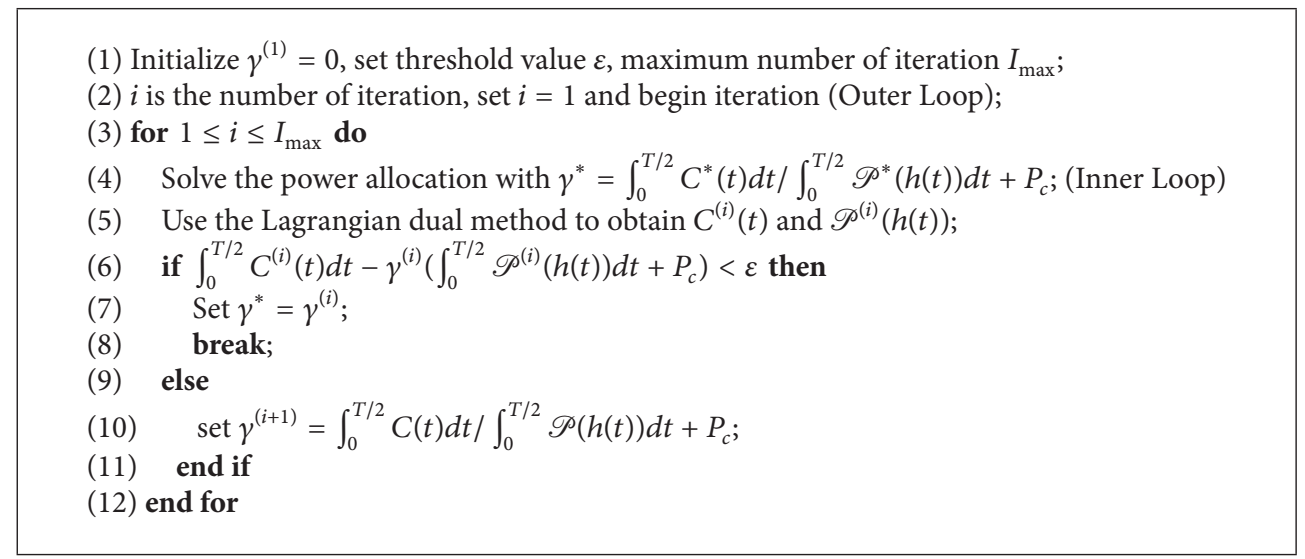

Algorithm 1: Energy-efficient power allocation.

Problem 4 (optimal resource allocation in the inner loop).

$$
\begin{aligned}
& \text { (P4) } \max _{\mathscr{P}} \int_{0}^{T / 2} C^{(i)}(t) d t \\
& -\gamma^{(i)}\left(\int_{0}^{T / 2} \mathscr{P}^{(i)}(h(t)) d t+P_{c}\right) \\
& \text { subject to } \frac{2}{T} \int_{0}^{T / 2} C^{(i)}(t) d t \\
& \geq \lambda+\frac{1}{\tau}+\frac{1}{\tau^{2}(\mu-\lambda)-\tau}, \\
& \frac{2}{T} \int_{0}^{T / 2} \mathscr{P}^{(i)}(h(t)) d t \leq P_{\text {ave }},
\end{aligned}
$$

where $\gamma^{(i)}$ is the $i$-th iteration energy-efficient value.

3.4. Lagrangian Dual Method. Based on the proposed iterative algorithm, we have obtained the $i$-th iteration energyefficient value $\gamma^{(i)}$; then the Problem 1 becomes a convex problem; we can use Lagrangian dual method to solve the optimal power allocation results [30]. The Lagrangian function of objective function can be written as

$$
\begin{aligned}
L(\mathscr{P}(h(t)), v, \beta)= & \int_{0}^{T / 2} C(t) d t \\
& -\gamma^{*}\left(\int_{0}^{T / 2} \mathscr{P}(h(t)) d t+P_{c}\right) \\
& -v \int_{0}^{T / 2}(C(t)-c) d t \\
& +\beta \int_{0}^{T / 2}\left(\mathscr{P}(h(t))-P_{\mathrm{ave}}\right) d t
\end{aligned}
$$

where $v$ and $\beta$ are the Lagrange multipliers.
To maximize the Lagrangian function, we only need to maximize the power allocation pointwise.

$$
\begin{aligned}
L(\mathscr{P}(h(t)), v, \beta)= & C(t)-\gamma^{*}\left(\mathscr{P}(h(t))+P_{c}\right) \\
& -v(C(t)-c) \\
& +\beta\left(\mathscr{P}(h(t))-P_{\text {ave }}\right) \\
= & \log (1+h(t) \mathscr{P}(h(t))) \\
& -\gamma^{*}\left(\mathscr{P}(h(t))+P_{c}\right) \\
& -v(\log (1+h(t) \mathscr{P}(h(t)))-c) \\
& +\beta\left(\mathscr{P}(h(t))-P_{\text {ave }}\right) .
\end{aligned}
$$

And then, using the Lagrangian dual method differentiating $L(\mathscr{P}(h(t)), v, \beta)$ with respect to $\mathscr{P}(h(t))$ and setting the result to zero, we can calculate $\mathscr{P}(h(t))$.

$$
\begin{aligned}
\frac{\partial L(\mathscr{P}(h(t)), v, \beta)}{\partial \mathscr{P}(h(t))}= & \frac{h(t)}{\ln 2(1+h(t) \mathscr{P}(h(t)))}-\gamma^{*} \\
& -v \frac{h(t)}{\ln 2(1+h(t) \mathscr{P}(h(t)))} \\
& +\beta=0 . \\
\mathscr{P}(h(t))= & \frac{1-v}{\ln 2\left(\gamma^{*}-\beta\right)}-\frac{1}{h(t)} .
\end{aligned}
$$

(11) is the water filling form power allocation scheme. The power allocation requires accurate CSI. In HSR scenario, considering the moving track of the train is certain, we utilize the predicted path loss information instead of accurate CSI. Therefore, we call (11) as predictable path loss based time domain power allocation method (PPTPA).

Furthermore, since $C(t) \geq c$, we obtain

$$
\mathscr{P}(h(t))=\max \left\{\frac{1-v}{\ln 2\left(\gamma^{*}-\beta\right)}-\frac{1}{h(t)}, \frac{2^{c}-1}{h(t)}\right\} .
$$

We can calculate the value of $v$ and $\beta$ by using the bisection method. (12) is a kind of hybrid power allocation scheme 
based on PPTPA. We call it as QoE Constrained EE Power Allocation (QCEPA).

\section{Capacity Maximization with EE Constraint Problem}

EE performance is a main concern point for the design of wireless communication. However, if we only consider the EE maximization, the system capacity may degrade seriously $[20,32]$. In Section 3, we only considered the PISs. In practice, the communication system of HSR may bear not only the PISs but also the TCSs. In this section, we consider the communication system of HSR bears both of them and then design a hybrid power allocation scheme based on PPTPA to support more services subject to EE performance and transmission delay constraints.

4.1. Problem Formulation. From Section 3, we get the optimal EE performance subject to transmission delay and average power constraints. To analyze the EE performance and capacity tradeoff, we formulate the problem to maximize capacity subject to constraints on delay, average power, and EE requirement in this section.

Problem 5 (capacity maximization problem). Considering the transmission delay constraint, average power, and EE requirement, the capacity maximization problem in the downlink C-RAN of HSR can be formulated as

$$
\begin{aligned}
\text { (P5) } \max _{\mathscr{P}} & \int_{0}^{T / 2} C(t) d t \\
\text { subject to } & \frac{2}{T} \int_{0}^{T / 2} C(t) d t \geq c, \\
& c \geq \lambda+\frac{1}{\tau}+\frac{1}{\tau^{2}(\mu-\lambda)-\tau}, \\
& \frac{2}{T} \int_{0}^{T / 2} \mathscr{P}(h(t)) d t \geq P_{\mathrm{ave}}, \\
& \frac{\int_{0}^{T / 2} C(t) d t}{\int_{0}^{T / 2} \mathscr{P}(h(t)) d t+P_{c}} \geq \eta \gamma^{*},
\end{aligned}
$$

where $\eta$ is chosen as a weight of the optimal EE. $\gamma^{*}$ is the optimal EE value of Problem 1, which is calculated in Section 3. Constraints (13b)-(13d) have the same meaning with constraints $(4 \mathrm{~b})-(4 \mathrm{~d})$ in Problem 1. Constraint (13e) means that the EE performance is large or equal to the EE requirement.

\section{Constraint (13e) can be written as}

$$
\int_{0}^{T / 2} C(t) d t-\eta \gamma^{*}\left(\int_{0}^{T / 2} \mathscr{P}(h(t)) d t+P_{c}\right) \geq 0 .
$$

It becomes a convex constraint. Intuitively, objective function is a convex function, constraint (13b) and constraint (13c) are all convex constraints. Therefore, Problem 5 is a convex optimization problem.
4.2. Lagrangian Dual Method. Problem 5 is a convex optimization problem, so it can be solved by classical convex optimization methods. In this section, we use Lagrangian dual method to solve Problem 5. The Lagrangian function of objective function can be written as

$$
\begin{aligned}
& L(\mathscr{P}(h(t)), v, \beta, \mu)=\int_{0}^{T / 2} C(t) d t \\
& \quad-v \int_{0}^{T / 2}(C(t)-c) d t \\
& \quad+\beta \int_{0}^{T / 2}\left(\mathscr{P}(h(t))-P_{\mathrm{ave}}\right) d t-\mu\left(\int_{0}^{T / 2} C(t) d t\right. \\
& \left.\quad-\eta \gamma^{*}\left(\int_{0}^{T / 2} \mathscr{P}(h(t)) d t+P_{c}\right)\right),
\end{aligned}
$$

where $v, \beta$, and $\mu$ are the Lagrange multipliers.

As in Problem 3, our aim is to maximize the Lagrangian function, and we only need to maximize the power allocation pointwise.

$$
\begin{aligned}
L(\mathscr{P} & (h(t)), v, \beta, \mu) \\
= & C(t)-v(C(t)-c)+\beta\left(\mathscr{P}(h(t))-P_{\mathrm{ave}}\right) \\
& -\mu\left(C(t)-\eta \gamma^{*}\left(\mathscr{P}(h(t))+P_{c}\right)\right) \\
= & C(t)(1-v-\mu)+\left(\mu \eta \gamma^{*}+\beta\right) \mathscr{P}(h(t))+v c \\
& -\beta P_{\mathrm{ave}}+\mu \eta \gamma^{*} P_{c} .
\end{aligned}
$$

And then, using the Lagrangian dual method differentiating $L(\mathscr{P}(h(t)), v, \beta, \mu)$ with respect to $\mathscr{P}(h(t))$ and setting the result to zero, we can calculate $\mathscr{P}(h(t))$.

$$
\begin{aligned}
& \frac{\partial L(\mathscr{P}(h(t)), v, \beta, \mu)}{\partial \mathscr{P}(h(t))} \\
& \quad=(1-v-\mu) \frac{h(t)}{\ln 2(1+h(t) \mathscr{P}(h(t)))}+\mu \eta \gamma^{*}+\beta \\
& \quad=0 . \\
& \mathscr{P}(h(t))=\frac{v+\mu-1}{\ln 2\left(\mu \eta \gamma^{*}+\beta\right)}-\frac{1}{h(t)} .
\end{aligned}
$$

Furthermore, since $C(t) \geq c$, we get

$$
\mathscr{P}(h(t))=\max \left\{\frac{v+\mu-1}{\ln 2\left(\mu \eta \gamma^{*}+\beta\right)}-\frac{1}{h(t)}, \frac{2^{c}-1}{h(t)}\right\} .
$$

(18) is also a kind of hybrid power allocation scheme based on PPTPA. We call it as QoE Constrained Capacity Maximization Power Allocation (QCCMPA).

\section{Power Allocation Considering Small-Scale Fading}

In the previous sections, we only considered the influence of path loss on the channel. However, the fast time-varying 
small-scale fading is the characteristic of wireless channel of HSR; we need to consider the effect of small-scale fading on system performance. As we know, small-scale fading cannot be predicted accurately in HSR scenario. But the statistics information of small-scale fading can be known in the transmitter and it remains unchanged for a long time. Because the small-scale fading can reduce the system capacity, the EE performance can be reduced when we consider the smallscale fading. In this section, we evaluate the effect of smallscale fading on the energy-efficient power allocation policy.

The Nakagami-m model is used to describe the smallscale fading of HSR [29, 33]. $m$ is the fading factor, which increases from $1 / 2$ to $\infty$. The fading becomes Rayleigh when $m=1$.

We consider flat fading, and for narrowband signals, the received signal at time $t$ is

$$
y(t)=\sqrt{h_{s}(t) h(t)} x(t)+z(t), \quad 0 \leq t \leq \frac{2}{T},
$$

where $\sqrt{h_{s}(t)}$ is small-scale fading with Nakagami-m distribution. $z(t)$ is complex Gaussian noise with variance $\sigma^{2}$. The instantaneous channel capacity is

$$
C(t)=\log \left(1+h_{s}(t) h(t) \mathscr{P}(h(t))\right), \quad 0 \leq t \leq \frac{2}{T} .
$$

Therefore, the channel capacity in a half period is

$$
\int_{0}^{T / 2} C(t) d t=\int_{0}^{T / 2} \log \left(1+h_{s}(t) h(t) \mathscr{P}(h(t))\right) d t .
$$

In HSR scenario, we are interested in small delay constraint and large data arrival rate. The vertical distance $d_{0}$ and the coverage distance $d_{s}$ are small. In this case, the train can receive high SNR even when the train is on the edge of the cell [34]. Therefore, the channel capacity is

$$
\begin{aligned}
\int_{0}^{T / 2} C(t) d t \approx & \int_{0}^{T / 2} \log \left(h_{s}(t) h(t) \mathscr{P}(h(t))\right) d t \\
= & \int_{0}^{T / 2} \log (h(t) \mathscr{P}(h(t))) d t \\
& +\int_{0}^{T / 2} \log \left(h_{s}(t)\right) d t .
\end{aligned}
$$

From (22), we can see that the channel capacity would be reduced because of the factor $\int_{0}^{T / 2} \log \left(h_{s}(t)\right) d t$. Because the period that the train passes through one cell is much larger than the channel coherence time, the small-scale fading is ergodic. Therefore, we get

$$
\int_{0}^{T / 2} \log \left(h_{s}(t)\right) d t=E\left(\log \left(h_{s}(t)\right)\right) \frac{T}{2} .
$$

$\sqrt{h_{s}(t)}$ is Nakagami-m distribution with parameters $\Omega$ and $m$, where $\Omega$ is the average SNR and $m$ is the fading factor. Thus, we obtain

$$
E\left(\log \left(h_{s}(t)\right)\right)=\left[\psi(m)+\ln \frac{\Omega}{m}\right] \log e,
$$

where $\psi$ is Digamma function.
TABLE 1: Parameters in simulation.

\begin{tabular}{lcc}
\hline Parameter & Description & Value \\
\hline$d_{0}$ & Vertical distance & $50 \mathrm{~m}$ \\
$d_{s}$ & Coverage distance & $1500 \mathrm{~m}$ \\
$v$ & Train speed & $350 \mathrm{~km} / \mathrm{h}$ \\
$\alpha$ & Path loss exponent & 3 \\
$\mu$ & Service processing capacity & $6 \mathrm{bits} / \mathrm{s}$ \\
$\lambda$ & Data arrival rate & $5 \mathrm{bits} / \mathrm{s} / \mathrm{Hz}$ \\
$\tau$ & Delay constraint & $400 \mathrm{~ms}$ \\
$\Delta$ & Frequency compensation & $1 \mathrm{~dB}$ \\
$P_{\text {ave }}$ & Average power threshold & $40 \mathrm{w}$ \\
\hline
\end{tabular}

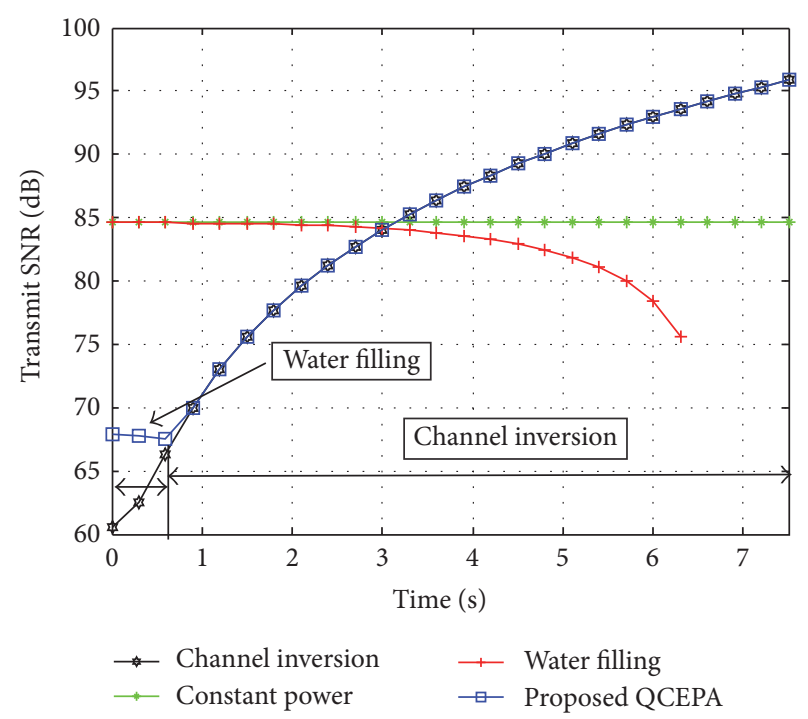

FIGURE 2: Transmit SNR of proposed QCEPA algorithm compared with other three different power allocation policies.

\section{Results and Discussions}

In this section, we provide emulation and extensive simulation results to validate previous theoretical analysis results. The simulation parameters are shown in Table 1.

6.1. EE Optimization Power Allocation Policy. We firstly evaluate the EE performance of the proposed QCEPA power allocation solution, and then we analyze the impact of the service processing capacity of VMs $\mu$, channel fading factor $m$, data arrival rate $\lambda$, and different train speeds $v$ on the EE performance. Finally, the effect of small-scale fading on the proposed QCEPA power allocation policy is evaluated.

In Figure 2, the proposed QCEPA algorithm is compared with other three different power allocation policies (water filling, channel inversion, and constant power allocation).

(i) Water filling power allocation: in this scheme, we compare the traditional water filling power allocation scheme. The objective function is to maximize the capacity with average power constraint. Compared to the proposed QCEPA, the traditional water filling 
power allocation does not consider the delay constraint, and the objective function is also different.

(ii) Channel inversion power allocation: in this scheme, the power allocation scheme is $\mathscr{P}(h(t))=\left(2^{c}-\right.$ 1) $/ h(t)$.

(iii) Constant power allocation: in this scheme, the power is constant in each time slot.

From Figure 2, we can see that the process of QCEPA is divided into two phases; from $0 \mathrm{~s}$ to $0.79 \mathrm{~s}$ is the first phase, the channel condition is good, and water filling power allocation policy is used; from $0.79 \mathrm{~s}$ to $7.5 \mathrm{~s}$ is the second phase, the channel condition becomes bad as the train approaches the cell edge, and RRU adopts channel inversion power allocation policy. It also can be seen that the water filling power allocation scheme is no power allocation when the time is $6.5 \mathrm{~s}$. The reason is that the channel condition is bad when the train is at the edge of the cell; the RRU does not allocate power to the passengers. From this figure, it also can be seen that the proposed QCEPA is different from the traditional water filling power allocation. The reason can be explained as follows. Because the channel condition is good from $0 \mathrm{~s}$ to $0.79 \mathrm{~s}$, QCEPA adopts the form of water filling power allocation to meet the delay constraint and achieve the maximum EE performance. From $0.79 \mathrm{~s}$ to $7.5 \mathrm{~s}$, because the channel condition is bad, QCEPA adopts channel inversion power allocation to meet the delay constraint and achieve the maximum EE performance. However, for the traditional water filling power allocation, because it does not consider the delay constraint and its objective function is to maximize capacity, the allocation results of QCEPA and the traditional water filling power allocation are different. Although these two algorithms are all the form of water filling, the values are different. For example, the solution of QCEPA is $\mathscr{P}(h(t))=\max \left\{(1-v) / \ln 2\left(\gamma^{*}-\right.\right.$ $\left.\beta)-1 / h(t),\left(2^{c}-1\right) / h(t)\right\}$, but the solution of traditional water filling is $\mathscr{P}(h(t))=\max \{1 /-\lambda \ln 2-1 / h(t), 0\}$.

Figure 3 illustrates the EE performance versus different train speeds under different power allocation policies. From this figure we can see that when the train speed is faster, the EE performance is worse. This is because that the average channel quality of the high-speed train is worse than the low speed train in unit time. It also can be seen that the EE performance of proposed algorithm outperforms the other three power allocation schemes. When the train speed is $350 \mathrm{~km} / \mathrm{h}$, the EE performance can reach $0.3408 \mathrm{bps} / \mathrm{Hz} / \mathrm{W}$. And the EE performance of channel inversion power allocation policy is the worst. This is because the channel inversion power allocation policy tries to keep a constant transmission rate, no matter whether the channel condition is good or bad. When the channel condition is not very good, the RRU has to increase transmit power, but the channel capacity increases inconspicuously. Therefore, the EE performance becomes bad.

Service preprocessing capability of VMs $\mu$ can affect the transmission time and further affect the EE performance. Therefore, $\mu$ is an indirect factor affecting the EE performance. Figure 4 shows EE performance versus service processing capability of VMs under different $m$ factors. From this figure, as can be seen, the more serious small-scale fading

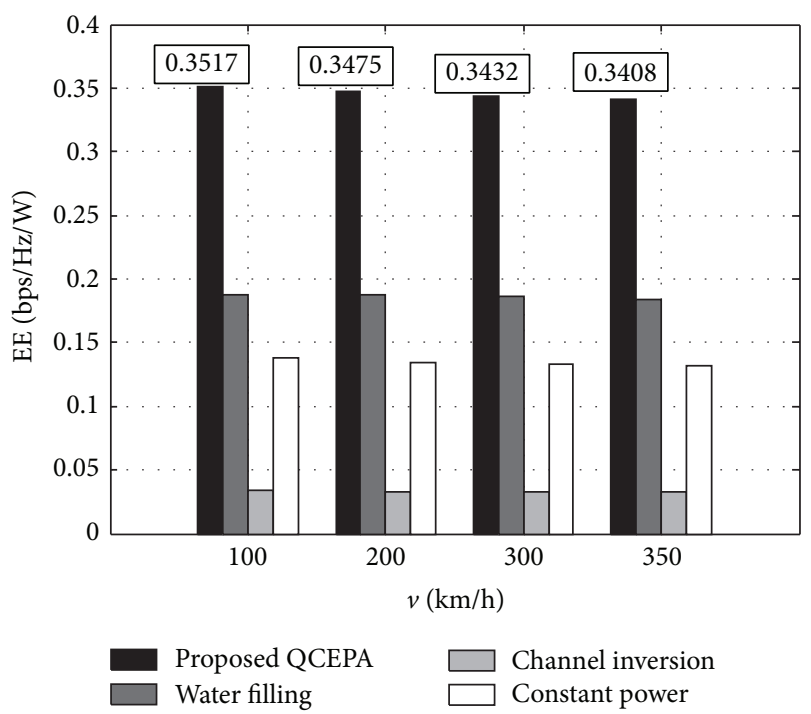

FIGURE 3: EE performance versus different train speeds under different power allocation policies.

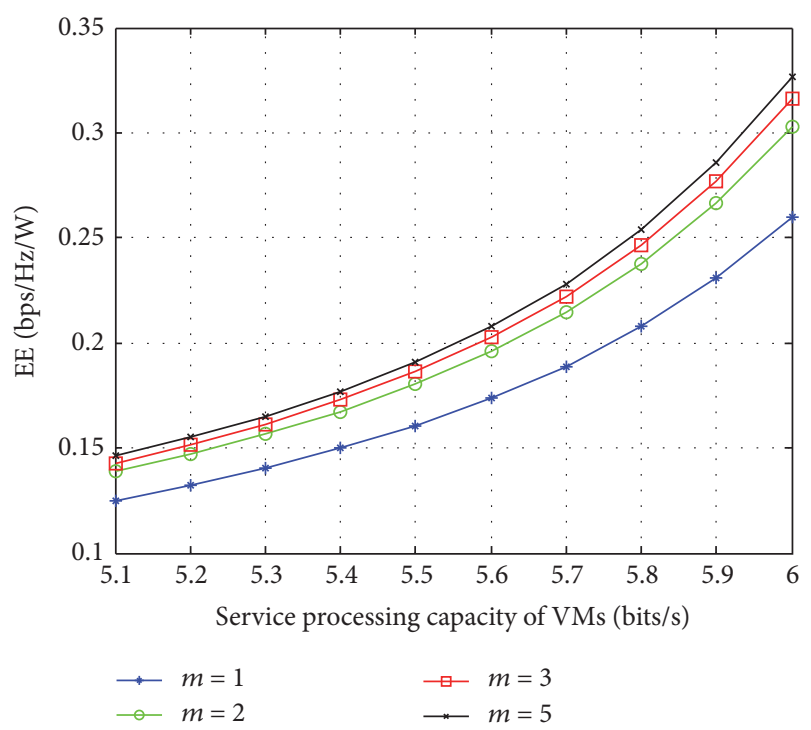

FIGURE 4: EE performance versus service processing capacity of VMs under different $m$ factors.

leads to worse EE performance. Also we can see that with the increasing service processing capability of VMs, the EE performance also increases. We can explain as follows: when the service processing capability is stronger, the service time is shorter, so the RRU has more time to transmit the data as the channel condition becomes good, which leads to better EE performance.

Another affecting EE performance factor is data arrival rate $\lambda$. Figure 5 illustrates $\mathrm{EE}$ performance versus data arrival rate with considering small-scale fading or not. In Algorithm 1, we only consider the effect of large-scale fading on power allocation scheme. However, the small-scale fading effect cannot be eliminated. Therefore, we must evaluate the effect of small-scale fading on the power allocation results. In 


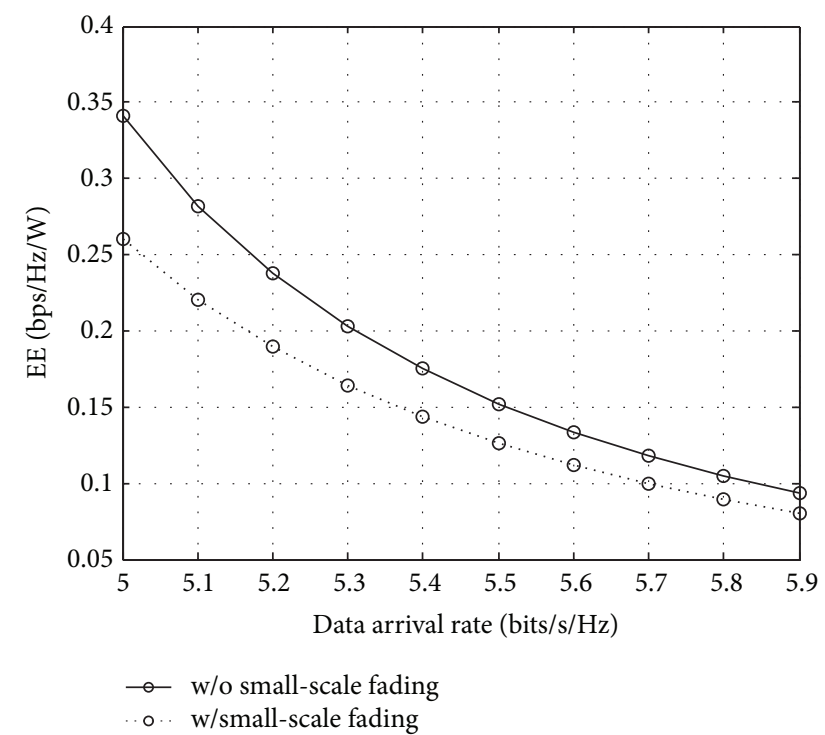

FIGURE 5: EE performance versus data arrival rate with considering small-scale fading when $m=1$ or not.

Section 5, we analyze the effect of small-scale fading on EE performance. For this figure, our goal is to show the effect of small-scale fading on EE performance. It can be seen that with the data arrival rate increases, the EE performance decreases. This is because when the data arrival rate gets higher, the RRU needs more power to increase the transmission rate, so the EE performance becomes bad. Furthermore, the EE performance is obviously decreased when the small-scale fading is considered. It is because that the channel prediction is not accurate, which leads to the unprecise power allocation policy. However, from Figure 5 we can see that the EE performance only decreases by $19.3 \%$ when the small-scale fading is considered. Therefore, the power allocation policy is still effective.

6.2. Maximum Capacity Power Allocation Policy. In this section, we evaluate the proposed QCCMPA power allocation scheme and then analyze the impact of small-scale fading on the capacity.

Figure 6 illustrates transmit SNR of proposed QCCMPA algorithm compared with other three different power allocation policies when $\eta=95 \%$. The process of QCCMPA is also divided into two phases; water filling power allocation is used in the first phase, and it takes longer time than that in Figure 2; channel inversion power allocation is used in the second phase. The form of QCCMPA is the same as QCEPA in Figure 2. However, the transmission power and the duration of each phase are different. This is because that the objective function of Problem 5 is to maximize capacity. If we want to get larger capacity, we need take more time to adopt the water filling power allocation scheme. From Figure 6 we can see that the power value in first phase of QCCMPA is larger than that in first phase of QCEPA. This is because the channel condition is good in the first phase, and we increase the transmission power to get larger capacity

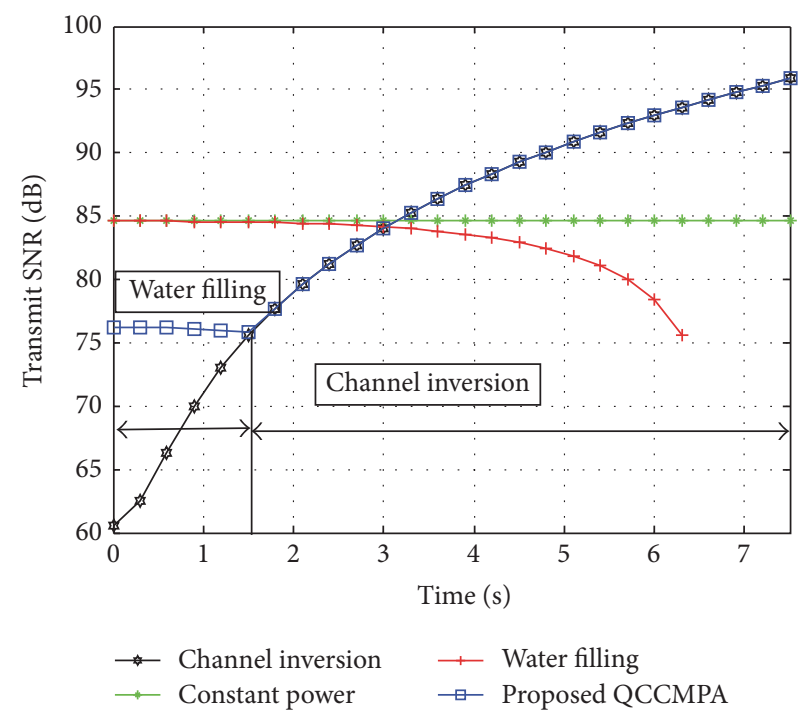

FIgURE 6: Transmit SNR of proposed QCCMPA algorithm compared with other three different power allocation policies when $\eta=$ $95 \%$.

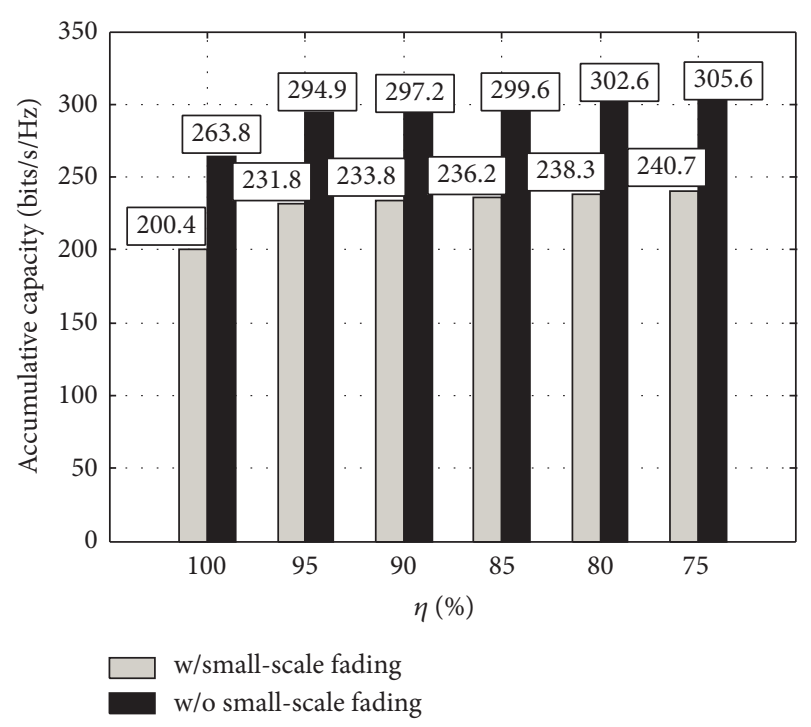

Figure 7: Capacity under different EE performance ratio requirements with considering small-scale fading or not.

in this phase. However, as the train moves away from the cell center, the channel condition becomes bad. If we also increase transmission power in the second phase, the channel capacity increases very slightly but the EE performance decreases seriously, which cannot satisfy our requirements. Therefore, to increase system capacity, the best solution is to take more time to adopt the water filling power allocation scheme when the channel condition is good. From this figure, it also can be seen that the proposed QCCMPA is different from the traditional water filling power allocation, which can be explained for the same reason as Figure 2.

Figure 7 illustrates the accumulative capacity under different EE performance ratio requirements with considering 


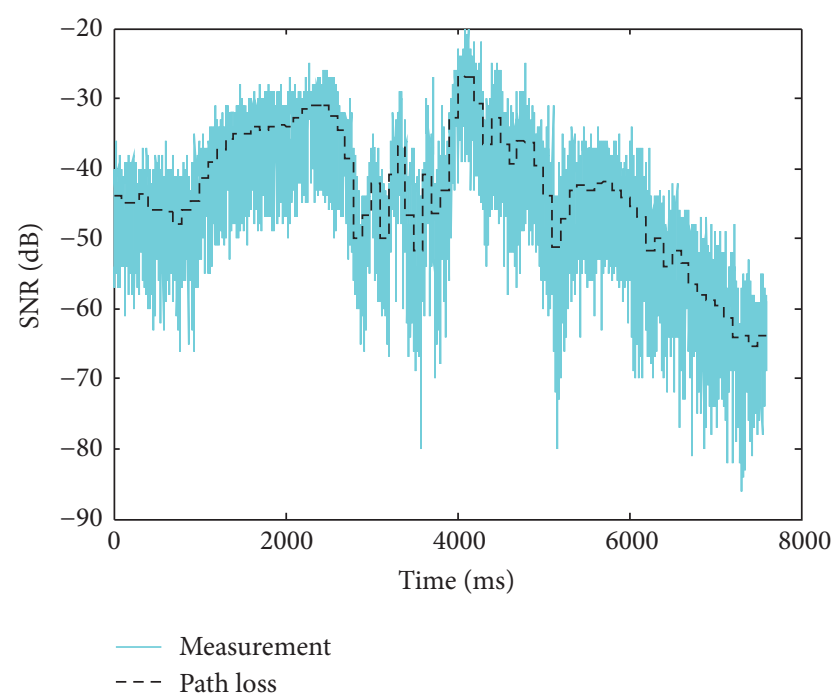

Figure 8: The measured path loss information.

small-scale fading or not. As can be seen, when the EE performance reduces from $100 \%$ to $95 \%$, the capacity is increased by $11.76 \%$ compared to $5 \% \mathrm{EE}$ loss. However, when the EE performance reduces from $100 \%$ to $75 \%$, the capacity only increases by $15.84 \%$. In practical scenarios, we can make a balance between EE and capacity according to our requirements. In Figure 7, we also consider small-scale fading with $m=1$, where the EE performance reduces from $100 \%$ to $95 \%$, and the capacity increases by about $15.66 \%$. When EE performance ratio reduces to $75 \%$ of the optimal value, the capacity increases by about $20.12 \%$. Therefore, the power allocation scheme is also effective when considering small-scale fading.

6.3. Performance Emulation. In order to evaluate the effectiveness of the proposed PPTPA in the real HSR scenario, we utilize the channel measurement of Zhengzhou-Xian HSR line [33] to perform it. The channel measurement and path loss information are shown in Figure 8.

We utilize the measured path loss and small-scale fading values to emulate the EE performance versus data arrival rate. And we compare the theoretical analysis with the measured data. Figure 9 has the same trend with Figure 5 and it can be explained for the same reasons. In Figure 5, the EE performance of theoretical analysis decreases by $19.3 \%$ when the small-scale fading is considered. However, in Figure 9 the EE performance only decreases by $15.81 \%$ when we utilize the measured values. In addition, the EE performance with measured small-scale fading is between $m=2$ and $m=$ 3. Therefore, the proposed power allocation algorithm is effective.

\section{Conclusion}

In this paper, we investigated two problems in C-RAN of HSR. Because the train location can be predicted, we utilized the path loss information to simplify the CSI of the train and proposed PPTPA to solve two problems. The first problem

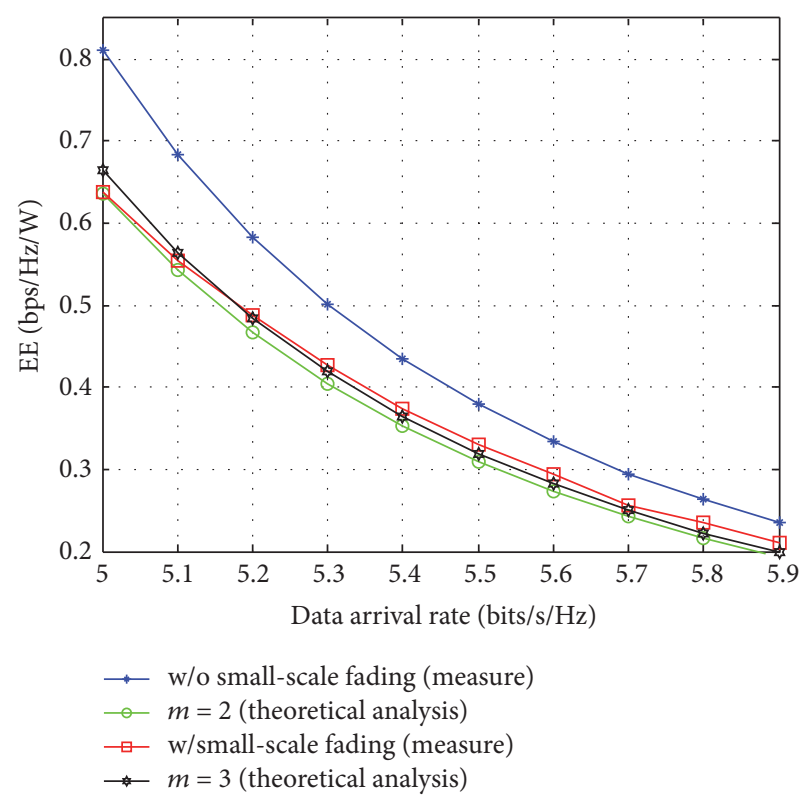

FIGURE 9: EE performance versus data arrival rate with measured values and theoretical analysis.

is the energy-efficient power allocation problem with delay constraint when the communication system of HSR only bears PISs. The second problem is the capacity optimization with joint $\mathrm{EE}$ and services transmission delay constraints when the communication system of HSR bears PISs and TCSs. The effect of small-scale fading on the proposed power allocation schemes was evaluated. Emulation results and extensive simulation results based on Zhengzhou-Xian HSR line channel measurements trace have demonstrated that proposed two power allocation policies can achieve EE maximization and capacity maximization. For the first problem, compared with water filling scheme, the EE performance of proposed algorithm is improved by $85 \%$. For the capacity maximization with $\mathrm{EE}$ constraint problem, the capacity in the proposed scheme can be increased by $11.76 \%$, when the EE performance reduces from $100 \%$ to $95 \%$.

\section{Appendix}

Proof of Lemma 2. We prove Lemma 2 with two steps as follows [25].

We prove the sufficient condition of Lemma 2 firstly. We define $\gamma^{*}$ as the optimal energy-efficient value of Problem $1, \gamma^{*}=\int_{0}^{T / 2} C^{*}(t) d t / \int_{0}^{T / 2} \mathscr{P}^{*}(h(t)) d t+P_{\mathcal{c}}$, where $\mathscr{P}^{*}(h(t))$ is the optimal power allocation policy and $C^{*}(t)$ is the corresponding channel capacity. Obviously, $\gamma^{*}$ should satisfy

$$
\begin{aligned}
\gamma^{*} & =\frac{\int_{0}^{T / 2} C^{*}(t) d t}{\int_{0}^{T / 2} \mathscr{P}^{*}(h(t)) d t+P_{c}} \\
& \geq \frac{\int_{0}^{T / 2} C(t) d t}{\int_{0}^{T / 2} \mathscr{P}(h(t)) d t+P_{c}} .
\end{aligned}
$$


From (A.1), we can get

$$
\begin{array}{r}
\int_{0}^{T / 2} C(t) d t-\gamma^{*}\left(\int_{0}^{T / 2} \mathscr{P}(h(t)) d t+P_{c}\right) \leq 0, \\
\int_{0}^{T / 2} C^{*}(t) d t-\gamma^{*}\left(\int_{0}^{T / 2} \mathscr{P}^{*}(h(t)) d t+P_{c}\right)=0 .
\end{array}
$$

Therefore, we can conclude $\max _{\mathscr{P}} \int_{0}^{T / 2} C(t) d t \quad-$ $\gamma^{*}\left(\int_{0}^{T / 2} \mathscr{P}(h(t)) d t+P_{c}\right)=0$. The sufficient condition is proved.

Secondly, we prove the necessary condition. Suppose that $\widehat{\mathscr{P}}^{*}(h(t))$ is the optimal power allocation policy of the reformulated objective function and $\widehat{C}^{*}(t)$ is the corresponding channel capacity. Therefore, $\int_{0}^{T / 2} \widehat{C}^{*}(t) d t-$ $\gamma^{*}\left(\int_{0}^{T / 2} \widehat{\mathscr{P}}^{*}(h(t)) d t+P_{c}\right)=0$. For a feasible power allocation policy $\mathscr{P}(h(t))$ and corresponding channel capacity $C(t)$, they can be written as

$$
\begin{aligned}
& \int_{0}^{T / 2} C(t) d t-\gamma^{*}\left(\int_{0}^{T / 2} \mathscr{P}(h(t)) d t+P_{c}\right) \\
& \quad \leq \int_{0}^{T / 2} \widehat{C}^{*}(t) d t-\gamma^{*}\left(\int_{0}^{T / 2} \widehat{\mathscr{P}}^{*}(h(t)) d t+P_{c}\right) \\
& \quad=0 .
\end{aligned}
$$

From above inequality, we can get

$$
\begin{gathered}
\frac{\int_{0}^{T / 2} C(t) d t}{\int_{0}^{T / 2} \mathscr{P}(h(t)) d t+P_{c}} \leq \gamma^{*}, \\
\frac{\int_{0}^{T / 2} \widehat{C}(t) d t}{\int_{0}^{T / 2} \widehat{\mathscr{P}}(h(t)) d t+P_{c}}=\gamma^{*} .
\end{gathered}
$$

Therefore, the optimal power allocation policy $\widehat{\mathscr{P}}^{*}(h(t))$ for the reformulated objective function is also the optimal power allocation policy for the original function. The necessary condition is proved.

\section{Disclosure}

This paper has been presented in part at the IEEE 83rd Vehicular Technology Conference (VTC spring) [35], Nanjing, China, 15-18 May 2016.

\section{Competing Interests}

The authors declare that they have no competing interests.

\section{Acknowledgments}

This work was partly supported by the Fundamental Research Funds for the Central Universities (no. 2015RC032), the State Key Laboratory of Rail Traffic Control and Safety (nos. RCS2015K011 and RCS2015ZT001), the Key Laboratory of Wireless Sensor Network and Communication, Chinese Academy of Sciences (no. 2013005), the National Natural Science Foundation of China (nos. 61501023, U1334202, and U1534201), and the Project of China Railway Corporation under Grant no. 2016X003-O.

\section{References}

[1] B. Ai, X. Cheng, T. Kurner et al., "Challenges toward wireless communications for high-speed railway," IEEE Transactions on Intelligent Transportation Systems, vol. 15, no. 5, pp. 2143-2158, 2014.

[2] S. Xu, G. Zhu, C. Shen, Y. Lei, and Z. Zhong, "Analysis and optimization of resource control in high-speed railway wireless networks," Mathematical Problems in Engineering, vol. 2014, Article ID 781654, 13 pages, 2014.

[3] J. Wang, H. Zhu, and N. J. Gomes, "Distributed antenna systems for mobile communications in high speed trains," IEEE Journal on Selected Areas in Communications, vol. 30, no. 4, pp. 675-683, 2012.

[4] C-RAN: The Road Green RAN, China Mobile Research Institute, 2011.

[5] M. Behjati, M. H. Alsharif, R. Nordin, and M. Ismail, "Energy efficient and high capacity tradeoff in distributed antenna system for a green cellular network," Journal of Computer Networks and Communications, vol. 2015, Article ID 170854, 9 pages, 2015.

[6] I. Chih-Lin, C. Rowell, S. Han, Z. Xu, G. Li, and Z. Pan, “Toward green and soft: a $5 \mathrm{G}$ perspective," IEEE Communications Magazine, vol. 52, no. 2, pp. 66-73, 2014.

[7] D. Feng, C. Jiang, G. Lim, L. J. Cimini Jr., G. Feng, and G. Y. Li, "A survey of energy-efficient wireless communications," IEEE Communications Surveys and Tutorials, vol. 15, no. 1, pp. 167178, 2013.

[8] G. Fettweis and E. Zimmermann, "ICT energy consumptiontrends and challenges," in Proceedings of the 11th International Symposium on Wireless Personal Multimedia Communications (WPMC '08), pp. 2006-2009, 2008.

[9] M. De Sanctis, E. Cianca, and V. Joshi, "Energy efficient wireless networks towards green communications," Wireless Personal Communications, vol. 59, no. 3, pp. 537-552, 2011.

[10] A. González-Gil, R. Palacin, P. Batty, and J. P. Powell, "A systems approach to reduce urban rail energy consumption," Energy Conversion and Management, vol. 80, pp. 509-524, 2014.

[11] A. González-Gil, R. Palacin, and P. Batty, "Optimal energy management of urban rail systems: key performance indicators," Energy Conversion and Management, vol. 90, pp. 282-291, 2015.

[12] A. Dahane, A. Loukil, B. Kechar, and N.-E. Berrached, "Energy efficient and safe weighted clustering algorithm for mobile wireless sensor networks," Mobile Information Systems, vol. 2015, Article ID 475030, 18 pages, 2015.

[13] L. Kong, X.-Y. Liu, M. Tao et al., "Resource-efficient data gathering in sensor networks for environment reconstruction," Computer Journal, vol. 58, no. 6, pp. 1330-1343, 2014.

[14] L. Kong and X. Liu, "MZig: enabling multi-packet reception in ZigBee," in Proceedings of the 21st Annual International Conference on Mobile Computing and Networking (MobiCom '15), pp. 552-565, Paris, France, September 2015. 
[15] G. P. Fettweis, K.-G. Chen, and R. Tafazoli, "Green radio: energy efficiency in wireless networks," Journal of Communications and Networks, vol. 12, no. 2, pp. 99-102, 2010.

[16] Y. Zhou and W. Yu, "Optimized backhaul compression for uplink cloud radio access network," IEEE Journal on Selected Areas in Communications, vol. 32, no. 6, pp. 1295-1307, 2014.

[17] Y. Shi, J. Zhang, and K. B. Letaief, "Group sparse beamforming for green cloud-RAN," IEEE Transactions on Wireless Communications, vol. 13, no. 5, pp. 2809-2823, 2014.

[18] S.-H. Park, O. Simeone, O. Sahin, and S. Shamai, "Robust and efficient distributed compression for cloud radio access networks," IEEE Transactions on Vehicular Technology, vol. 62, no. 2, pp. 692-703, 2013.

[19] M. Peng, K. Zhang, J. Jiang, J. Wang, and W. Wang, "Energyefficient resource assignment and power allocation in heterogeneous cloud radio access networks," IEEE Transactions on Vehicular Technology, vol. 64, no. 11, pp. 5275-5287, 2015.

[20] L. Musavian and Q. Ni, "Effective capacity maximization with statistical delay and effective energy efficiency requirements," IEEE Transactions on Wireless Communications, vol. 14, no. 7, pp. 3824-3835, 2015.

[21] X. Li, X. Ge, X. Wang, J. Cheng, and V. C. M. Leung, "Energy efficiency optimization: joint antenna-subcarrier-power allocation in OFDM-DASs," IEEE Transactions on Wireless Communications, vol. 15, no. 11, pp. 7470-7483, 2016.

[22] K. Xie, X. Huang, S. Hao, M. Ma, P. Zhang, and D. Hu, "E ${ }^{3}$ MC: improving energy efficiency via elastic multi-controller sdn in data center networks," IEEE Access, vol. 4, pp. 6780-6791, 2016.

[23] Q. Wu, W. Chen, D. W. Kwan Ng, J. Li, and R. Schober, "Usercentric energy efficiency maximization for wireless powered communications," IEEE Transactions on Wireless Communications, vol. 15, no. 10, pp. 6898-6912, 2016.

[24] C. C. Zarakovitis and Q. Ni, "Maximizing energy efficiency in multiuser multicarrier broadband wireless systems: convex relaxation and global optimization techniques," IEEE Transactions on Vehicular Technology, vol. 65, no. 7, pp. 5275-5286, 2016.

[25] D. W. K. Ng, E. S. Lo, and R. Schober, "Energy-efficient resource allocation in OFDMA systems with large numbers of base station antennas," IEEE Transactions on Wireless Communications, vol. 11, no. 9, pp. 3292-3304, 2012.

[26] X. Yang, X. Li, B. Ning, and T. Tang, "A survey on energyefficient train operation for urban rail transit," IEEE Transactions on Intelligent Transportation Systems, vol. 17, no. 1, pp. 2-13, 2016.

[27] J. Tang, W. P. Tay, and T. Q. S. Quek, "Cross-layer resource allocation with elastic service scaling in cloud radio access network," IEEE Transactions on Wireless Communications, vol. 14, no. 9, pp. 5068-5081, 2015.

[28] P. Demestichas, A. Georgakopoulos, D. Karvounas et al., " $5 \mathrm{G}$ on the Horizon: key challenges for the radio-access network," IEEE Vehicular Technology Magazine, vol. 8, no. 3, pp. 47-53, 2013.

[29] C. Zhang, P. Fan, K. Xiong, and P. Fan, "Optimal power allocation with delay constraint for signal transmission from a moving train to base stations in high-speed railway scenarios," IEEE Transactions on Vehicular Technology, vol. 64, no. 12, pp. 5775-5788, 2015.

[30] S. Boyd and L. Vandenberghe, Convex Optimization, Cambridge University Press, 2004.

[31] M. Peng, Y. Li, J. Jiang, J. Li, and C. Wang, "Heterogeneous cloud radio access networks: a new perspective for enhancing spectral and energy efficiencies," IEEE Wireless Communications, vol. 21, no. 6, pp. 126-135, 2014.
[32] L. Musavian and Q. Ni, "Delay-QoS-driven spectrum and energy efficiency tradeoff," in Proceedings of the 1st IEEE International Conference on Communications (ICC '14), pp. 4981-4986, Sydney, Australia, June 2014.

[33] S. Lin, L. Kong, L. He et al., "Finite-state Markov modeling for high-speed railway fading channels," IEEE Antennas and Wireless Propagation Letters, vol. 14, pp. 954-957, 2015.

[34] S. Lin, Z. Zhong, L. Cai, and Y. Luo, "Finite state Markov modelling for high speed railway wireless communication channel," in Proceedings of the IEEE Global Communications Conference (GLOBECOM '12), pp. 5421-5426, IEEE, Anaheim, Calif, USA, December 2012.

[35] S. Li, G. Zhu, S. Lin, Q. Gao, S. Xu, and L. Xiong, "Energyefficient power allocation in cloud radio access network of high-speed railway," in Proceedings of the 83rd IEEE Vehicular Technology Conference (VTC Spring '16), Nanjing, China, May 2016. 

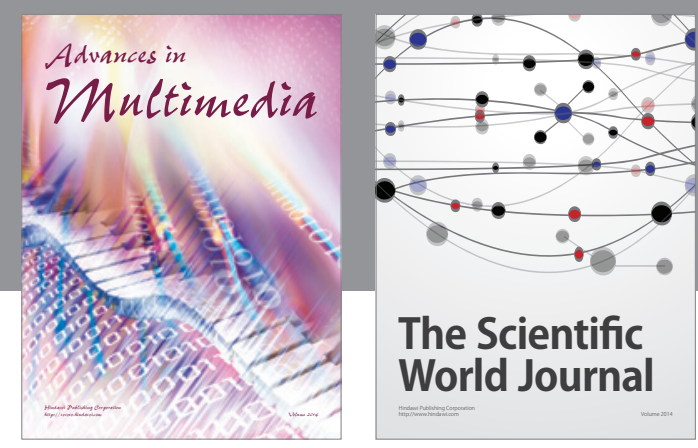

The Scientific World Journal
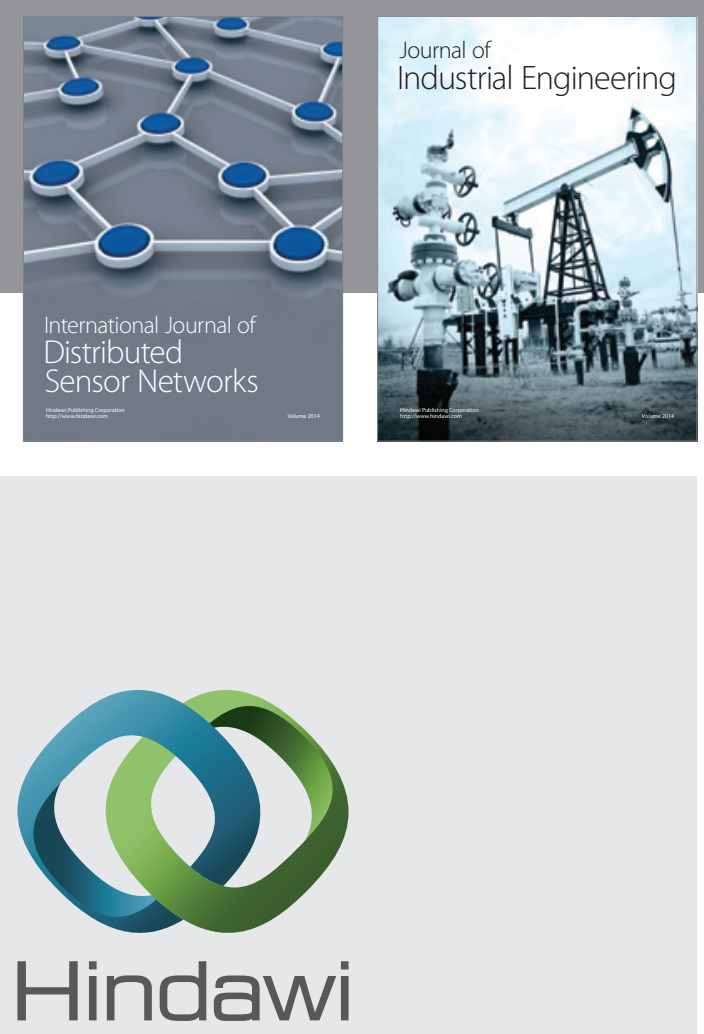

Submit your manuscripts at

https://www.hindawi.com

\section{Computer Networks} and Communications
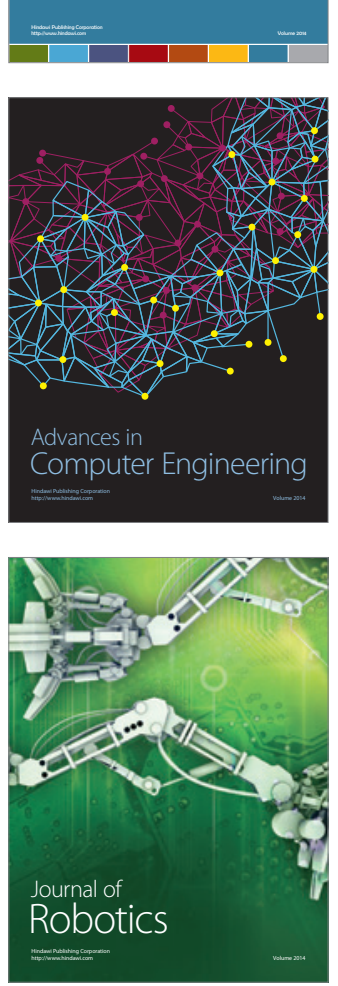
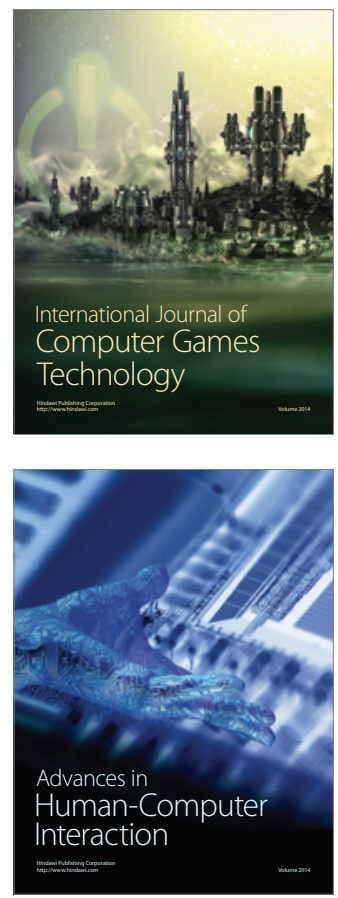
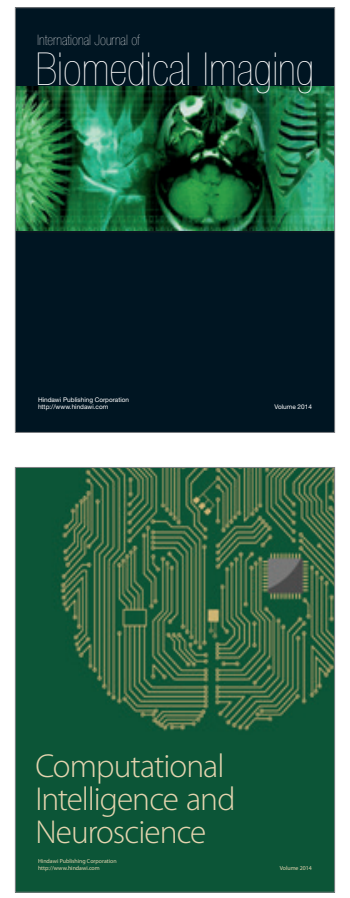
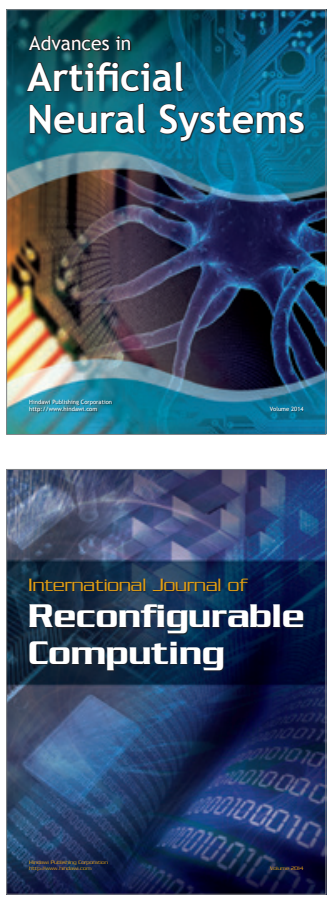
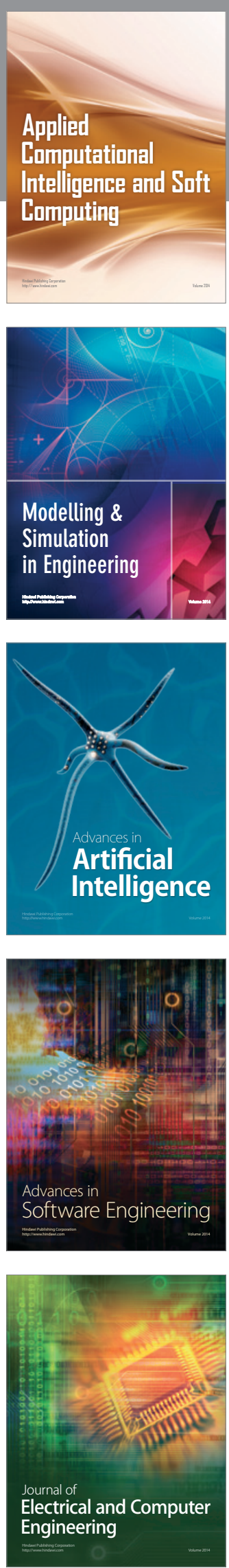